Europhysics Letters

PREPRINT

\title{
Surface relaxation of lyotropic lamellar phases
}

\author{
H. BARY-SOROKER ${ }^{1}$ and H. DiAMANT ${ }^{2}$ \\ 1 School of Physics \&3 Astronomy \\ 2 School of Chemistry \\ Raymond 83 Beverly Sackler Faculty of Exact Sciences, Tel Aviv University, Tel Aviv \\ 69978, Israel
}

PACS. 61.30.St - Lyotropic phases.

PACS. 68.03. Kn - Gas-liquid and vacuum-liquid interfaces, dynamics (capillary waves).

PACS. 82.70.Uv - Surfactants, micellar solutions, vesicles, lamellae, amphiphilic systems.

\begin{abstract}
We study the relaxation modes of an interface between a lyotropic lamellar phase and a gas or a simple liquid. The response is found to be qualitatively different from those of both simple liquids and single-component smectic-A liquid crystals. At low rates it is governed by a non-inertial, diffusive mode whose decay rate increases quadratically with wavenumber, $|\omega|=A q^{2}$. The coefficient $A$ depends on the restoring forces of surface tension, compressibility and bending, while the dissipation is dominated by the so-called slip mechanism, i.e., relative motion of the two components of the phase parallel to the lamellae. This surface mode has a large penetration depth which, for sterically stabilised phases, is of order $\left(d q^{2}\right)^{-1}$, where $d$ is the microscopic lamellar spacing.
\end{abstract}

Lyotropic lamellar phases occupy large portions in the phase diagrams of amphiphilic molecules (surfactants) in solution [1,2]. They consist of stacks of parallel fluid membranes separated by microscopic ( 1-10 nm thick) layers of solvent [fig. 1(a)], thus having the symmetry of a smectic-A liquid crystal [3]. These phases appear in numerous applications, e.g., in the cosmetic and detergent industries. Lamellar bodies are found also in biological systems such as the lung [4]. Apart from the free surfaces of lamellar phases with air, surfactant phase diagrams contain also large coexistence regions (so-called immiscibility gaps) [1], in which a lamellar phase has an equilibrium interface with an isotropic liquid such as a dilute micellar solution or an $L_{3}$ (sponge) phase [1,2]. Spherical lamellar structures in the form of multilamellar vesicles (onions) dispersed in a solvent are commonly encountered as well [5]. Thus, the surface response of these phases is a fundamental issue relevant to a large variety of experimental systems. The static response of smectics to surface deformations was thoroughly studied $[3,6,7]$. The dynamics of surface perturbations in thermotropic (single-component) smectics were investigated as well, for both semi-infinite systems and finite films [8-16]. In the current Letter we analyse the surface relaxation of lyotropic (two-component) lamellar phases and demonstrate the essentially different surface dynamics of this ubiquitous class of materials.

Surface modes characterise the relaxation dynamics of surface perturbations whose amplitude decays with increasing distance into the bulk material [fig. 1(a)]. In a simple liquid,

(C) EDP Sciences 
a)

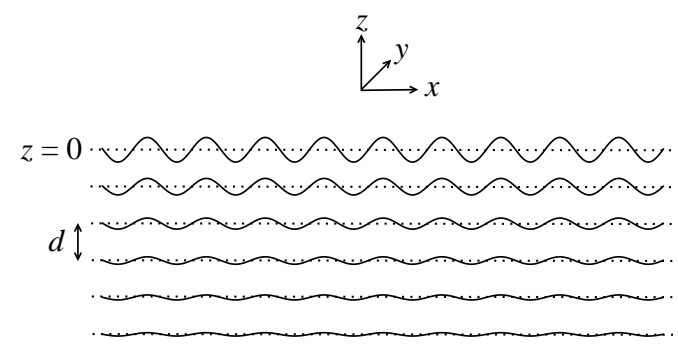

b)

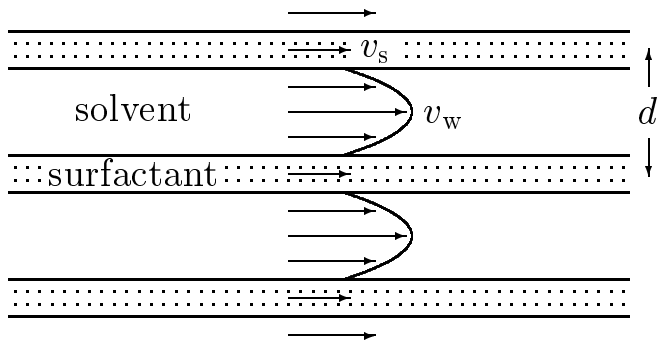

Fig. 1 - a) Schematic view of the system. A semi-infinite lamellar phase occupies the region $z<0$, having an interface at $z=0$ with a gas or a simple liquid. A surface perturbation is shown, whose amplitude decays to zero at $z \rightarrow-\infty$. b) Visualisation of the slip flow, in which the two components of the lamellar phase have different average velocities.

having mass density $\rho$, viscosity $\eta$ and surface tension $\gamma$, the surface dynamics depend on two dimensionless numbers: the Reynolds number, Re $\equiv \rho|\omega| /\left(\eta q^{2}\right)$, and the Capillary number, $\mathrm{Ca} \equiv \eta|\omega| /(\gamma|q|)$, where $q$ and $\omega$ are the perturbation wavenumber and frequency, respectively. If inertia is neglected, one is left with the Capillary number alone, leading to a decay rate $|\omega| \sim \gamma|q| / \eta$. However, substituting this result back in the requirement $\operatorname{Re} \ll 1$ yields $|q| \gg \rho \gamma / \eta^{2}$. Thus, e.g., for water such a non-inertial, overdamped response is restricted to wavelengths much smaller than $10^{-2} \mu \mathrm{m}$, while larger-wavelength perturbations are governed by inertial capillary waves [17], whose dispersion relation is set by $\mathrm{ReCa} \sim 1$.

The viscoelastic hydrodynamics of smectic-A liquid crystals [3] were formulated in the seminal work of ref. [18]. Due to the anisotropy of these materials the viscosity tensor contains five independent coefficients which, for incompressible flows, can be reduced to three, denoted by $\eta_{\mathrm{M}}, \eta_{\mathrm{T}}$ and $\eta_{\mathrm{V}}$ [19]. While $\eta_{\mathrm{M}}$ is related to relative sliding of layers, and is therefore comparable to the viscosity of the solvent (water) in lyotropic phases, the other two are associated with distortions of the membranes themselves and, hence, are comparable to the overall apparent viscosity of the liquid, which is $2-3$ orders of magnitude larger $[19,20]$. The elasticity is characterised by a compression modulus $B$ and a bending modulus $K$. These elastic moduli define two Deborah numbers in addition to the Capillary and Reynolds numbers. The dynamics thus depend on four dimensionless numbers, which we define as

$$
\operatorname{De}_{B} \equiv \eta_{\mathrm{M}}|\omega| / B, \quad \operatorname{De}_{K} \equiv \eta_{\mathrm{M}}|\omega| /\left(K q^{2}\right), \quad \mathrm{Ca} \equiv \eta_{\mathrm{M}}|\omega| /(\gamma|q|), \quad \operatorname{Re} \equiv \rho|\omega| /\left(\eta_{\mathrm{M}} q^{2}\right)
$$

(More accurately, in the case of lyotropic phases one should consider separately, depending on the frequency, two layer-compression moduli - the modulus at constant surfactant concentration (for high frequencies) and the modulus at constant surfactant chemical potential (low frequencies), which is typically much smaller [21]. Our main focus here is on the slow response for which $B$ should be regarded, unless otherwise noted, as the smaller, fixed-chemical-potential modulus.)

The surface modes of a semi-infinite thermotropic smectic phase were studied in ref. [14]. Despite the much higher viscosity of these phases compared to ordinary simple liquids (water), the conclusion of this work was that the low-frequency surface modes were inertial, elastic (Rayleigh) waves of second sound, their dispersion relation being set by $\operatorname{ReDe}_{B} \sim 1$. In a detailed analysis of low-Reynolds-number surface modes of thermotropic smectics [22], we have reached a similar conclusion, namely, that non-inertial relaxation is restricted to small (sub-micron) wavelengths. On the other hand, it is hard to envisage the surface dynamics 
of a small lamellar droplet or an onion (of, say, $10-10^{2} \mu \mathrm{m}$ scale), whose inter-layer spacings and surroundings are pervaded by viscous solvent, as dominated by underdamped inertial waves. The disagreement between this intuition and the aforementioned results suggests that lyotropic lamellar phases may be dominated by another dissipative mechanism, different from the usual viscous one.

The hydrodynamic theory of smectics was extended to lyotropic phases in refs. [19,23, 24]. Brochard and de Gennes [19] were the first to recognise that, unlike isotropic binary mixtures, the spatial organisation of the two components in a lamellar phase allows for collective motion of one component relative to the other parallel to the layers [fig. 11(b)]. This bulk slip mode was observed experimentally [23,25-28]. We show in the current work that the additional dissipation due to slip has profound consequences for the surface dynamics. The dominance of this dissipative mechanism can be demonstrated by the following simple argument (to be made rigourous later on). The transport coefficient associated with slip, $\mu$, relates to the mobility of a Poiseuille flow of solvent between two membranes [fig. 1(b)], $\mu \sim d^{2} / \eta_{\mathrm{W}}$, where $d$ is the inter-membrane spacing and $\eta_{\mathrm{W}}$ the solvent viscosity [19]. The resulting friction force per unit volume is $\sim \mu^{-1} v, v$ being a characteristic relative flow velocity, while the force density arising from viscous stresses is $\sim \eta_{\mathrm{T}, \mathrm{V}} q^{2} v$. Hence, the slip dissipation dominates for $q \ll\left(\eta_{\mathrm{T}, \mathrm{V}} \mu\right)^{-1 / 2} \sim(10 d)^{-1}$ — a condition satisfied for all physically relevant wavelengths.

The system under consideration is schematically depicted in fig. 1(a). A semi-infinite lamellar phase occupies the region $z<0$, the layers lying, on average, parallel to the $x y$ plane. At $z=0$ there is an interface between the lamellar phase and a dilute gas or a simple liquid. We use Latin indices $(i, j)$ to denote vector components along all three axes $(x, y, z)$ and Greek indices $(\alpha, \beta)$ for components along the two lateral directions $(x, y)$.

The hydrodynamic description of thermotropic smectics requires six scalar hydrodynamic fields $[3,18]$. Assuming an isothermal, incompressible flow, one is left with only three independent fields, e.g., two of the three components of the flow velocity $v_{i}(\mathbf{r}, t)$ at position $\mathbf{r}$ and time $t$, and an additional scalar field, $u(\mathbf{r}, t)$, defining the local displacement of the layers from their equilibrium position. Three dynamic modes result: two second-sound waves and a transverse shear (vorticity) diffusive mode. In lyotropics another scalar field is required [19, 23], e.g., the local surfactant volume fraction. As a result, another mode emerges, i.e., the slip (baroclinic) mode. In writing the hydrodynamic equations we use the variables $v_{i}(\mathbf{r}, t), u(\mathbf{r}, t)$, and a pressure field $p(\mathbf{r}, t)$ (of which, we recall, only three are independent). To include the slip degree of freedom in lyotropics another velocity field is added [19], which we take as the lateral velocity of the surfactant component, $v_{\mathrm{s}, \alpha}(\mathbf{r}, t)$. Velocity differences between the two components, $v_{\mathrm{s}, \alpha}-v_{\alpha}$, are driven by in-plane stresses in the membranes [19]. We focus here on the simplest treatment, where the membranes are considered as incompressible two-dimensional (2D) liquids with no $2 \mathrm{D}$ viscous stresses. (This assumption is also consistent with the limit, mentioned above, of the fixed-chemical-potential compression modulus being much smaller than the fixed-concentration one.) In this description the driving force for slip is provided solely by a lateral pressure gradient, $\partial_{\alpha} \psi(\mathbf{r}, t)$. Out of the three new variables, $v_{\mathrm{s}, \alpha}$ and $\psi$, similarly, only one is independent.

In terms of the aforementioned variables, the hydrodynamic equations for a lamellar phase take the form

$$
\begin{aligned}
& \rho\left(\partial_{t} v_{i}+v_{j} \partial_{j} v_{i}\right)=\partial_{j} \sigma_{i j} \\
& \partial_{i} v_{i}=0 \\
& \partial_{\alpha} v_{\mathrm{s}, \alpha}=0 \\
& v_{\mathrm{s}, \alpha}-v_{\alpha}=-\mu \partial_{\alpha} \psi
\end{aligned}
$$




$$
\partial_{t} u-v_{z}=0 .
$$

Equation (2) is the Navier-Stokes equation, where $\sigma_{i j}$, to be specified below, is the stress tensor. Equations (3) and (4) impose mass conservation on the incompressible overall flow and 2D surfactant flow, respectively. Equation (5) asserts a linear relation between the slip velocity and its driving force. In eq. (6) we have assumed that permeation of material perpendicular to the layers is negligible [3]. We write the stress tensor as $\sigma=\sigma^{\mathrm{r}}+\sigma^{\mathrm{d}}$, where the "reactive" part is given by $[19,29]$

$$
\sigma_{x x}^{\mathrm{r}}=\sigma_{y y}^{\mathrm{r}}=-p-\psi+K \partial_{\beta \beta z} u, \quad \sigma_{z z}^{\mathrm{r}}=-p+B \partial_{z} u, \quad \sigma_{\alpha z}^{\mathrm{r}}=-K \partial_{\beta \beta \alpha} u, \quad \sigma_{x y}^{\mathrm{r}}=0,
$$

and the dissipative part by [19]

$$
\sigma_{\alpha \beta}^{\mathrm{d}}=\eta_{\mathrm{T}}\left(\partial_{\alpha} v_{\beta}+\partial_{\beta} v_{\alpha}\right), \quad \sigma_{z z}^{\mathrm{d}}=2 \eta_{\mathrm{V}} \partial_{z} v_{z}, \quad \sigma_{\alpha z}^{\mathrm{d}}=\eta_{\mathrm{M}}\left(\partial_{\alpha} v_{z}+\partial_{z} v_{\alpha}\right) .
$$

At $z=0$ the tangent force per unit area, $\sigma_{\alpha z}$, must change continuously across the interface, whereas the perpendicular one, $\sigma_{z z}$, has a discontinuity due to surface tension. If the surface is free (the other phase is a dilute gas), these conditions reduce to

$$
z=0: \quad \sigma_{\alpha z}=0, \quad \sigma_{z z}=\gamma \partial_{\alpha \alpha} u
$$

Since we deal with surface perturbations, the bulk far away from the interface is assumed to remain at equilibrium,

$$
z \rightarrow-\infty: \quad v_{i}=v_{\mathrm{s}, \alpha}=u=0 .
$$

If the phase in the region $z>0$ is a liquid, its hydrodynamic equations should be added, and the boundary conditions at $z=0$ are modified. Since the introduction of a simple liquid leads to a minor effect [22], we shall briefly comment on it below and otherwise focus on the simpler case of a free surface.

We are concerned with small deviations from equilibrium and, therefore, the nonlinear term in eq. (2) can be neglected. Equations (2)-(10) then define a closed set of linear equations with the appropriate boundary conditions, from which the surface modes can be derived. For simplicity we restrict the discussion to variations in the $x$ and $z$ directions only. Substituting a perturbation of the form $f(x, z, t) \propto e^{i q x-i \omega t}$, where $f$ stands for any of the hydrodynamic variables, we obtain a 4 th-order equation for $v_{z}$,

$$
\partial_{z z z z} v_{z}-\left(S+\Theta-2-\operatorname{De}_{B}^{-1}-\operatorname{Re}\right) q^{2} \partial_{z z} v_{z}+\left(1-\operatorname{De}_{K}^{-1}-\operatorname{Re}\right) q^{4} v_{z}=0 .
$$

In eq. (11) the dynamic numbers $\left(\mathrm{De}_{B}, \mathrm{De}_{K}, \mathrm{Re}\right)$ are as defined in eq. (1) with $|\omega|$ replaced by $i \omega$, and $S$ and $\Theta$ are two additional dimensionless parameters, the former associated with slip, $S \equiv\left(\eta_{\mathrm{M}} \mu q^{2}\right)^{-1}$, and the latter with the viscosity anisotropy, $\Theta \equiv 2\left(\eta_{\mathrm{T}}+\eta_{\mathrm{V}}\right) / \eta_{\mathrm{M}} \gg 1$. The solution to eq. (11) is given by

$$
\begin{aligned}
v_{z} & =\left(C_{+} e^{\alpha_{+} z}+C_{-} e^{\alpha_{-} z}\right) e^{i q x-i \omega t}, \quad \alpha_{ \pm}=|q|\left[\left(S+\Theta-2-\operatorname{De}_{B}^{-1}-\operatorname{Re} \pm \Gamma^{1 / 2}\right) / 2\right]^{1 / 2} \\
\Gamma & \equiv\left(S+\Theta-2-\operatorname{De}_{B}^{-1}-\operatorname{Re}\right)^{2}-4\left(1-\operatorname{De}_{K}^{-1}-\operatorname{Re}\right),
\end{aligned}
$$

where the two spatial decay coefficients, $\alpha_{ \pm}$, have been chosen as the ones with a positive real part so as to satisfy the boundary conditions at $z \rightarrow-\infty$, eq. (10). Imposing the interfacial boundary conditions (9) yields two linear equations for the amplitudes $C_{+}$and $C_{-}$, whose determinant is set to zero to find the dispersion relation, $\omega=\omega(q)$. The resulting equation is

$$
\mathrm{Ca}^{-1}\left(\alpha_{+}+\alpha_{-}\right) /|q|+\operatorname{Re}-\left(1-\mathrm{De}_{K}^{-1}-\mathrm{Re}\right)^{1 / 2}\left(S+\Theta-\mathrm{De}_{B}^{-1}-\mathrm{De}_{K}^{-1}-\mathrm{Re}\right)=0,
$$


where $\mathrm{Ca}$ is as defined in eq. (11) with the replacement $|\omega| \rightarrow i \omega$.

Equation (13), together with the expressions for $\alpha_{ \pm}$given in eq. (12), can be solved for $\omega$ only numerically. However, we can significantly simplify the problem by noticing the following. First, the slip mechanism will dominate over the viscous one when $S \gg \Theta$. As already discussed above, this amounts to a condition for $q$ which is practically always valid. Secondly, for typical lamellar phases the length $\lambda=(K / B)^{1 / 2}$ is very small (of order a few $d$ ) and, as a result, $\operatorname{De}_{B}^{-1} \gg \operatorname{De}_{K}^{-1}$ for any relevant $q$. We also take $\operatorname{Re} \ll \operatorname{De}_{K}^{-1}<1, \Theta \ll \operatorname{De}_{B}^{-1}$, and $S>$ $\mathrm{De}_{B}^{-1}$. (The self-consistency of these conditions will be later verified.) Under these assumptions we find $\alpha_{+} /|q| \simeq\left(S-\mathrm{De}_{B}^{-1}\right)^{1 / 2} \gg \alpha_{-} /|q| \simeq\left[\left(1-\mathrm{De}_{K}^{-1}\right) /\left(S-\mathrm{De}_{B}^{-1}\right)\right]^{1 / 2}$. Consequently, eq. (13) is simplified to

$$
\mathrm{Ca}^{-1}-\left[\left(1-\mathrm{De}_{K}^{-1}\right)\left(S-\mathrm{De}_{B}^{-1}\right)\right]^{1 / 2}=0,
$$

whose solution is

$$
\omega=-i A q^{2}, \quad A=\left[B \mu+K / \eta_{\mathrm{M}}+\sqrt{\left(B \mu-K / \eta_{\mathrm{M}}\right)^{2}+4 \mu \gamma^{2} / \eta_{\mathrm{M}}}\right] / 2 .
$$

Equation (15) is our central result. It describes a decay rate of a diffusive mode, which is affected by all three restoring forces and the slip dissipative mechanism. To make these results more transparent, let us specialise to sterically stabilised lamellar phases, where $B \sim$ $\left(k_{\mathrm{B}} T\right)^{2} /\left(\kappa d^{3}\right), K \sim \kappa / d$, and $\gamma \sim k_{\mathrm{B}} T / d^{2}, k_{\mathrm{B}} T$ being the thermal energy and $\kappa$ the bending modulus of a single membrane, typically a few $k_{\mathrm{B}} T$. (The low tension values in lyotropic phases, $\gamma \sim k_{\mathrm{B}} T / d^{2}$, arise from the diffusive contact of the surface membranes with membranes in the bulk, which are essentially tensionless.) Recalling that $\eta_{\mathrm{M}} \mu \sim d^{2}$, we find for such phases that all the terms appearing in eq. (15) are of the same order and $A \sim k_{\mathrm{B}} T /\left(\eta_{\mathrm{M}} d\right)$. This yields $10^{2}-10^{3} \mu \mathrm{m}^{2} / \mathrm{s}$ for $d=10 \mathrm{~nm}$ and $\eta_{\mathrm{M}}=10^{-2}$ poise. The spatial decay coefficients in this case are $\alpha_{+} \sim d^{-1}$ and $\alpha_{-} \sim d q^{2}$. Thus, the surface mode contains a boundary layer of microscopic thickness $\sim d$, and a deeply penetrating undulation whose thickness is much larger than the wavelength. The amplitude ratio of these two parts is $C_{+} / C_{-} \simeq-\alpha_{-}^{2} / q^{2}$, which is a small (negative) number, i.e., the dominant part is the deeply penetrating one.

Upon substitution of the results in the initial assumptions, their validity is readily confirmed. In particular, the Reynolds number is $\operatorname{Re} \sim \rho k_{\mathrm{B}} T /\left(\eta_{\mathrm{M}}^{2} d\right) \sim 10^{-4}-10^{-3}$ for the above parameters and $\rho=1 \mathrm{~g} / \mathrm{cm}^{3}$, irrespective of $q$. In the case of stiffer (electrostatically stabilised) phases where $B \gg\left(k_{\mathrm{B}} T\right)^{2} /\left(\kappa d^{3}\right)$, however, the assumption $\operatorname{Re} \ll \mathrm{De}_{K}^{-1}$ is satisfied only if $B \ll(K / \rho)^{1 / 2} / \mu$. For typical parameters this implies $B \ll 10^{9} \mathrm{erg} / \mathrm{cm}^{3}$. Hence, for very stiff lamellar phases inertia may become important, and one should return to the more general equations (12) and (13).

Static surface deformations of smectics have a large penetration depth as well, $\left(\lambda q^{2}\right)^{-1} \sim$ $\left(d q^{2}\right)^{-1}[6,7]$. Thus, the static and dynamic penetrations turn out to be similarly deep despite their different physical origins. While in the static case the large depth arises from the fact that the layers are much more resistant to compression than to bending, in the dynamic case it stems from the high resistance to relative motion of the two components compared with the viscous resistance to their overall flow. Once a deeply penetrating undulation exists, a thin boundary layer must appear as well to satisfy the surface boundary condition for the transverse stress, $\sigma_{\alpha z}=0$. If the boundary layer were absent, the ratio between the two transverse-stress terms would be $\partial_{\alpha} v_{z} / \partial_{z} v_{\alpha} \sim q^{2} / \alpha_{-}^{2} \gg 1$, whereas in the presence of a boundary layer of thickness $\alpha_{+}^{-1}$ we have $\partial_{\alpha} v_{z} / \partial_{z} v_{\alpha} \sim\left(q^{2} / \alpha_{+}^{2}\right)\left(C_{-} / C_{+}\right) \sim q^{4} /\left(\alpha_{+} \alpha_{-}\right)^{2} \sim 1$, thus allowing the transverse-stress contributions to balance one another.

It should be noted that, having employed the assumptions of $2 \mathrm{D}$-incompressible membranes and negligible Re, we have suppressed the second-sound and vorticity-diffusion modes, leaving only one bulk mode (the slip, baroclinic one) of the four existing in bulk lyotropic phases. 
EUROPHYSICS LETTERS

Rayleigh waves, therefore, cannot emerge from this analysis. Such modes should exist in lyotropics as in thermotropics [14]. Their frequency domain, however, is much higher than that of the relaxation discussed here. For the two rates to be comparable, one must have $(B / \rho)^{1 / 2} q \sim A q^{2}$, i.e., $q^{-1} \sim(B \rho)^{1 / 2} \mu \sim 1-10 \mathrm{~nm}$. Hence, the time scales are well separated for any relevant $q$ and the two responses can safely be studied as decoupled. (The separation of time scales is even larger, in fact, since one should use for the fast second-sound response the much larger compression modulus at constant concentration [21].)

If the phase in the region $z>0$ is taken to be a simple liquid [22], the only change is that the term $\mathrm{Ca}^{-1}$ in eqs. (13) and (14) is replaced with $\left(\mathrm{Ca}^{-1}-2 \eta / \eta_{\mathrm{M}}\right), \eta$ being the viscosity of the simple liquid. Therefore, the simple liquid does not affect the above results so long as $\eta \ll$ $\gamma /(A|q|)$. For sterically stabilised lamellar phases, for example, this implies that only when the simple liquid is orders of magnitude more viscous than the solvent, $\eta \sim \eta_{\mathrm{M}} /(d|q|) \gg \eta_{\mathrm{M}}$, will it have an appreciable effect on the surface relaxation. This is yet another consequence of the dominance of slip dissipation.

To summarise, in contrast with simple liquids and thermotropic smectics, whose surface dynamics are governed by underdamped waves (capillary or Rayleigh waves, respectively), we have found that surfaces of lyotropic lamellar phases can relax via a much slower, overdamped diffusive mode over a wide range of wavelengths. The key ingredient underlying this qualitatively different behaviour is the presence of viscous solvent in between the membranes and the resulting relative slip of surfactant and solvent layers. The relaxation is remarkably slow due to the strong friction (small $\mu$ ) introduced by this motion. For example, in sterically stabilised phases perturbations of a micron-scale wavelength are predicted to decay with a rate of $\sim 10^{3} \mathrm{~s}^{-1}$. To our best knowledge, experiments concerning the surface modes of lamellar phases have not yet been performed. The predictions of this work should be verifiable in, e.g., dynamic scattering experiments.

In this study we have considered semi-infinite, flat lamellar phases. Our results should be valid for finite films and curved lamellar structures (e.g., onions) provided that their size is sufficiently large, or the wavelength is sufficiently small. This restriction, however, is particularly severe in the current case because of the deep penetration of the inferred surface mode. The onion radius, for example, should be much larger than $\alpha_{-}^{-1} \sim\left(d q^{2}\right)^{-1} \gg q^{-1}$. For a typical onion of radius $R \sim 10 \mu \mathrm{m}$ and $d \sim 1 \mathrm{~nm}$ the wavelength must be much smaller than $(R d)^{1 / 2} \sim 10^{-1} \mu \mathrm{m}$. An extension of the theory to finite films and curved surfaces is needed, therefore, to accurately account for the surface dynamics of such lamellar objects.

Finally, lamellar surfaces out of equilibrium have been long known to exhibit an intriguing instability involving multilayer finger-like structures (myelin figures) [30,31]. We hope that the elucidation of the relaxation of lamellar surfaces presented here will be instrumental also in the resolution of this long-standing puzzle.

$* * *$

We thank D. Andelman, L. Bary-Soroker, M. Cates, B. Davidovitch, S. Egelhaaf, and M. Kozlov for helpful discussions. This work was supported by the US-Israel Binational Science Foundation (2002271). H.D. acknowledges additional support from the Israeli Council of Higher Education (Alon Fellowship).

\section{REFERENCES}

[1] Evans D. F. and Wennerstöm H., The Colloidal Domain, 2nd edition (Wiley, New York) 1999. 
[2] Safran S. A., Statistical Thermodynamics of Surfaces, Interfaces, and Membranes (Westview Press, Boulder) 2003.

[3] De Gennes P.-G. and Prost J., The Physics of Liquid Crystals, 2nd edition (Oxford University Press, New York) 1993.

[4] Schmitz G. and Müller G., J. Lipid Res., 32 (1991) 1539.

[5] Roux D., in Soft and Fragile Matter, edited by Cates M. E. and Evans M. R. (IOP, Bristol) 2000.

[6] Durand G., C. R. Acad. Sci. B, 275 (1972) 629.

[7] Fournier J. B., J. Phys. II France, 6 (1996) 985.

[8] Orsay Group on Liquid Crystals, J. Phys. Colloq., 36 (1975) C1-305.

[9] Holyst R., Phys. Rev. A, 46 (1992) 6748.

[10] Poniewierski A., Holyst R., Price A. C., Sorensen L. B., Kevan S. D. and Toner J., Phys. Rev. E, 58 (1998) 2027.

[11] Price A. C., Sorensen L. B., Kevan S. D., Toner J., Poniewierski A. and Holyst R., Phys. Rev. Lett., 82 (1999) 755.

[12] Poniewiersini A. Holyst R., Price A. C. and Sorensen L. B., Phys. Rev. E, 59 (1999) 3048.

[13] Chen H.-Y. and Jasnow D., Phys. Rev. E, 57 (1998) 5639.

[14] Fedorov D. O., Romanov V. P. and Ul'yanov S. V., Phys. Rev. E, 62 (2000) 681.

[15] Romanov V. P. and Ul'yanov S. V., Phys. Rev. E, 63 (2001) 031706.

[16] Romanov V. P. and Ul'yanov S. V., Phys. Rev. E, 66 (2002) 061701.

[17] Landau L. D. and Lifshitz E. M., Fluid Mechanics, 2nd edition (Butterworth-Heinermann, Oxford) 1987.

[18] Forster D., Lubensky T. C., Martin P. C., Swift J. and Pershan P. S., Phys. Rev. Lett., 26 (1971) 1016. Martin P. C., Parodi O. and Pershan P. S., Phys. Rev. A, 6 (1972) 2401.

[19] Brochard F. and de Gennes P.-G., Pramana Suppl., 1 (1975) 1.

[20] Wunenburger A. S., Colin A., Colin T. and Roux D., Eur. Phys. J. E, 2 (2000) 277.

[21] Lubensky T. C., Prost J. and Ramaswamy S., J. Phys. France, 51 (1990) 933.

[22] Bary-Soroker H., MSc Thesis, Tel Aviv University (2005).

[23] Nallet F., Roux D. and Prost J., J. Phys. France, 50 (1989) 3147.

[24] Bruinsma R. and Rabin Y., Phys. Rev. A, 45 (1992) 994.

[25] Chan W. and Pershan P. S., Phys. Rev. Lett., 39 (1977) 1368.

[26] Nallet F., Roux D. and Prost J., Phys. Rev. Lett., 62 (1989) 276.

[27] Zhang C. Y., Sprunt S. and Litster J. D., Phys. Rev. E, 48 (1993) 2850.

[28] Cross B. and Crassous J., Eur. Phys. J. E, 14 (2004) 249.

[29] Landau L. D. and Lifshitz E. M., Theory of Elasticity, 3rd edition (Butterworth-Heinermann, Oxford) 1986.

[30] Warren P. B. and Buchanan M., Curr. Opin. Colloid Interface Sci., 6 (2001) 287, and references therein.

[31] Huang J.-R., Zou, L.-N. and Witten T. A., Eur. Phys. J. E, 18 (2005) 279. 